\title{
Kinematics of Selected Planck Galactic Cold Clumps
}

\author{
E. Varga-Verebélyi ${ }^{1}$, L. V. Tóth ${ }^{2}$, G. Marton ${ }^{1}$, D. Marshall ${ }^{3}$, \\ K. Dobashi ${ }^{4}$ and T. Shimoikura ${ }^{4}$ \\ ${ }^{1}$ Konkoly Observatory of the Hungarian Academy of Sciences, Budapest, Hungary \\ ${ }^{2}$ Department of Astronomy, Eötvös Loránd University, Budapest, Hungary \\ ${ }^{3}$ Laboratoire AIM, CEA-IRFU/CNRS/Universite Paris Diderot, CEA Saclay, France \\ ${ }^{4}$ Department of Astronomy and Earth Sciences, Tokyo Gakugei University, Tokyo
}

We have completed a kinematical analysis of 184 selected Planck Galactic Cold Clumps (PGCC) (Planck Collaboration, 2015) in order to understand better the stability of molecular clouds and the induced star formations. Most of our clumps are in the second quadrant of the Galaxy. For the investigation we used ${ }^{12} \mathrm{CO},{ }^{13} \mathrm{CO}, \mathrm{C}^{18} \mathrm{O}$ line observations which covered the 184 PGCC fully or partially. The majority of the data were observed with the Osaka $1.85 \mathrm{~m}$ telescope in Japan (Nishimura et al. 2015) and we also have some observations with the KOSMA $3 \mathrm{~m}$ telescope, from Switzerland (Kramer et al. 2000) and with the IRAM 30 m telescope, Spain (www.iram-institute.org).

Based on 65 different size CO spectral maps we prepared 184 set of average spectra and found 249 line components. For the 184 PGCC we determined the excitation temperatures and optical depth, the velocity $(\operatorname{grad}(\mathrm{v}))$ and linewidth $(\operatorname{grad}(\Delta \mathrm{v}))$ gradients, the velocity, and linewidth dispersions and investigated the number of YSOs inside the clumps. We also created larger scale velocity field maps using our observations and column density maps using the reduced Planck observations and estimated distance to 40 PGCC, which were previously undetermined in the catalogue of PGCC.

The median of the absolute value of the $\operatorname{grad}(\mathrm{v})$ in our sample is around $1.3 \mathrm{~km} \mathrm{~s}^{-1} \mathrm{pc}^{-1}$, which seems to be CO line (i.e. density) independent, while it is typical both to the ${ }^{12} \mathrm{CO}$ and ${ }^{13} \mathrm{CO}$. The Spearman's rank correlation (Zar, 2005) of the position angles of the gradient lines considering different $\mathrm{CO}$ isotopomers is 0.54 , which shows that the position angles are related, however the correlation is definitely not strong.

We investigated the correlation of the $\operatorname{grad}(\mathrm{v})$ and $\operatorname{grad}(\Delta \mathrm{v})$, and call their correlation positive while increasing $\operatorname{grad}(\mathrm{v})$ leads increasing $\operatorname{grad}(\Delta \mathrm{v})$, and negative if the change of $\operatorname{grad}(\mathrm{v})$ and $\operatorname{grad}(\Delta \mathrm{v})$ are inverse. The two type of linewidth behaviours occur with nearly equal probability, and the two-sample t-test we performed also verify our assumption that the data is drawn from populations with the same true variance.

Keywords. molecular data, interstellar medium, ISM: clouds, ISM: structure, ISM: kinematics and dynamics, ISM: molecules

\section{References}

Planck Collaboration, Ade, P. A. R., Aghanim, N., et al. 2015, arXiv:1502.01599

Nishimura, A., Tokuda, K., Kimura, K., et al. 2015, ApJS, 216, 18

Kramer, C., Beuther, H., Simon, R., Stutzki, J., \& Winnewisser, G. 2000, Imaging at Radio through Submillimeter Wavelengths, 217, 194

Zar, J. H. 2005, Spearman Rank Correlation, Encyclopedia of Biostatistics. 7.

http://www.iram-institute.org/ 